\title{
Nanometer-scale ablation with a table-top soft $x$-ray laser
}

\author{
G. Vaschenko, A. Garcia Etxarri, C. S. Menoni, and J. J. Rocca \\ NSF ERC for Extreme Ultraviolet Science and Technology and Department of Electrical and Computer Engineering, \\ Colorado State University, Fort Collins, Colorado 80523 \\ O. Hemberg and S. Bloom \\ JMAR Technologies Inc., San Diego, California 92127
}

W. Chao, E. H. Anderson, and D. T. Attwood

NSF ERC for Extreme Ultraviolet Science and Technology and Center for X-Ray Optics, Lawrence Berkeley National Laboratory, Berkeley, California 94720

Y. Lu and B. Parkinson

Department of Chemistry, Colorado State University, Fort Collins, Colorado 80523

Received July 20, 2006; accepted September 7, 2006; posted September 21, 2006 (Doc. ID 73214); published November 22, 2006

\begin{abstract}
Ablation of holes with diameters as small as $82 \mathrm{~nm}$ and very clean walls was obtained in poly(methyl methacrylate) focusing pulses from a Ne-like Ar $46.9 \mathrm{~nm}$ compact capillary-discharge laser with a freestanding Fresnel zone plate diffracting into third order. These results demonstrate the feasibility of using focused soft $\mathrm{x}$-ray laser beams for the direct nanoscale patterning of materials and the development of new nanoprobes. (C) 2006 Optical Society of America

OCIS codes: $140.3390,140.7240,310.3840$.
\end{abstract}

Laser ablation of nanoscale features is of interest for the fabrication of nanodevices and for the development of new nanoprobes. The size of the smallest ablated features is limited mainly by the wavelength of the laser emission and by heat diffusion. A number of techniques have been recently developed to produce ablation features with submicrometer size.$^{1-6}$ Taking advantage of the well-defined ablation threshold in materials, craters with sizes ranging from 0.7 to $1.2 \mu \mathrm{m}$ were achieved in silicon by use of nanosecond ultraviolet (UV) pulses. ${ }^{1}$ Ablation features of the order of $200 \mathrm{~nm}$ have been demonstrated using femtosecond laser pulses in the near-infrared ${ }^{2,3}$ and $\mathrm{UV}^{4}$ spectral regions. Patterning of even smaller features has been realized using the electric field enhancement created at the tip of an atomic force microscope (AFM) by focused femtosecond laser pulses ${ }^{5}$ or using optical fibers to create near-field effects. ${ }^{6}$

In this work, we demonstrate the feasibility of directly ablating sub-100 $\mathrm{nm}$ nanoscale holes using a focused soft x-ray laser beam. Very clean ablation craters in poly(methyl methacrylate) (PMMA) were obtained by focusing the $46.9 \mathrm{~nm}$ wavelength output from a table-top capillary discharge laser with a freestanding Fresnel zone plate (FZP). The smallest craters, $82 \mathrm{~nm}$ in diameter, were obtained by placing the sample near the third-order focal plane of the FZP. This high spatial resolution coupled with the very small absorption depth of the $46.9 \mathrm{~nm}$ wavelength light in most materials are attractive features to exploit for nanopatterning applications. When combined with spectroscopic tools, this high-resolution ablation technique will also enable the development of new surface nanoprobes based on techniques such as laser-induced breakdown spectroscopy.

Figure 1 schematically illustrates the experimental setup. The laser is a compact table-top size device that uses a fast electrical discharge in an Ar-filled capillary tube to produce a population inversion and amplification in the $46.9 \mathrm{~nm}$ transition of Ne-like ions $\left(26.4 \mathrm{eV}\right.$ photon energy). ${ }^{7,8}$ For the $18 \mathrm{~cm}$ long capillary used in this experiment, the laser produces $\sim 0.1 \mathrm{~mJ}$ pulses of $\approx 1.2 \mathrm{~ns}$ duration with a repetition rate of up to $10 \mathrm{~Hz}$. The freestanding FZP was manufactured by electron-beam lithography ${ }^{9}$ into a $200 \mathrm{~nm}$ thick nickel film attached to a Si frame. The FZP had a $0.5 \mathrm{~mm}$ diameter, an outermost zone width of $200 \mathrm{~nm}$, and a NA=0.12. Its Rayleigh-type spatial

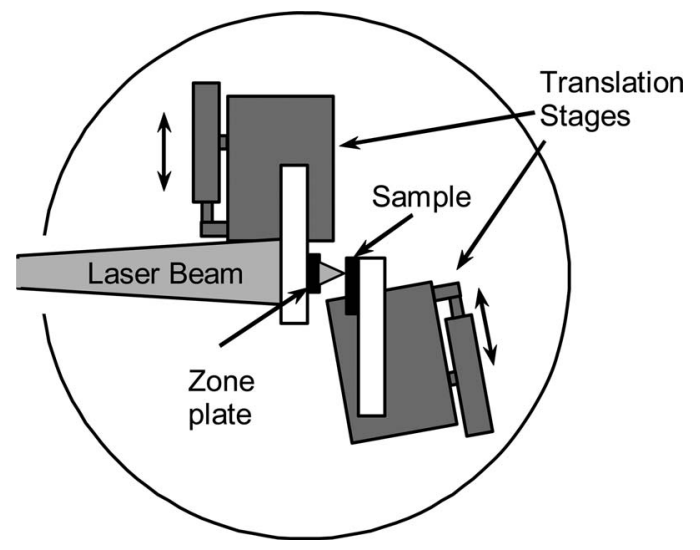

Fig. 1. Schematic of the soft x-ray laser ablation setup. 


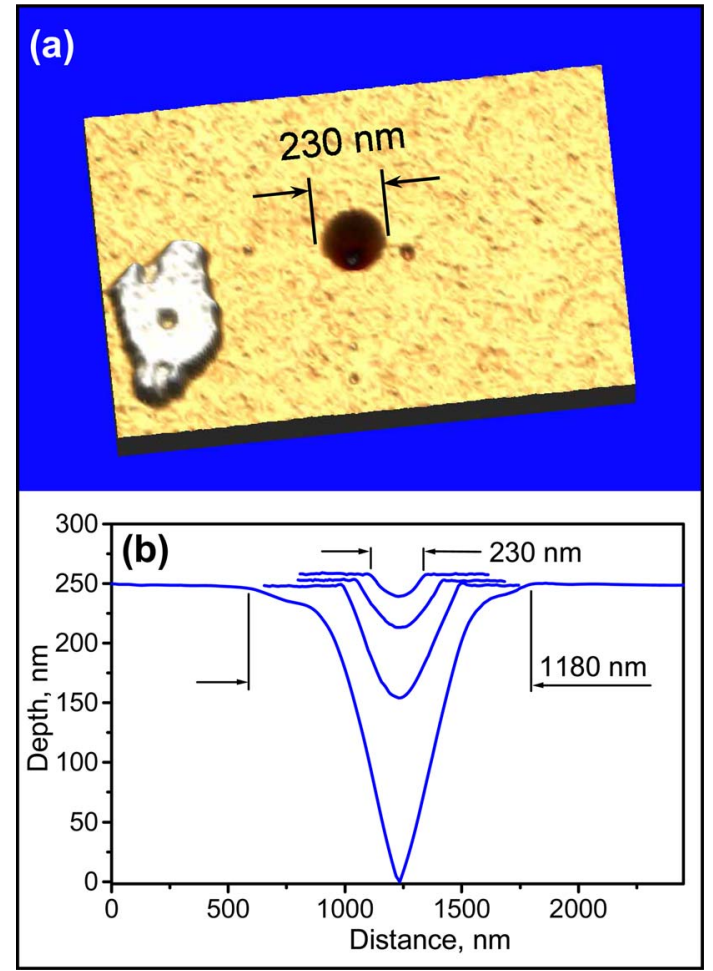

Fig. 2. (Color online) (a) AFM image of the ablation crater produced in PMMA using first diffraction-order focusing and $36 \times$ attenuation; (b) cross sections of AFM images of ablated craters obtained with attenuation factors of 1,6 , 14 , and $36 \times$.

resolution in the first diffraction order focus at $46.9 \mathrm{~nm}$ wavelength is $\sim 240 \mathrm{~nm} .{ }^{10}$ The FZP was mounted on an $X Y Z$ translation stage and positioned at $\sim 1.8 \mathrm{~m}$ from the laser exit where the laser beam has a FWHM diameter of $\sim 16 \mathrm{~mm}$. A laser pulse energy of $10 \mu \mathrm{J}$ was measured through a $6 \mathrm{~mm}$ pinhole placed at the location of the FZP using a vacuum photodiode. Considering that the FZP aperture is $0.5 \mathrm{~mm}$ in diameter and that its first diffraction order efficiency is $\sim 10 \%$, the energy delivered to the sample's surface is estimated to be $\sim 7 \mathrm{~nJ}$. The fluence onto the sample was controlled by introducing Ar gas into the vacuum chamber as a means of attenuating the laser beam following photoionization of the Ar atoms by the $24.6 \mathrm{eV}$ photons.

The sample consisted of a $500 \mathrm{~nm}$ layer of PMMA (PMMA-MicroChem, 950,000 molecular weight) spin coated on a $\mathrm{Si}$ wafer. The sample was positioned perpendicular to the incident soft x-ray laser beam, whereas the translation stage was tilted at an angle of $\sim 0.57^{\circ}$ with respect to the sample's surface to allow for precise positioning of the sample with respect to the zone plate focal plane (first-order depth of focus $\sim 3 \mu \mathrm{m})$. In this way, a $100 \mu \mathrm{m}$ transverse displacement of the sample corresponds to a $1 \mu \mathrm{m}$ displacement along the beam axis. The ablation craters produced by the $\lambda=46.9 \mathrm{~nm}$ laser beam were analyzed with a VEECO NanoScope III AFM used in a tapping mode with a $10 \mathrm{~nm}$ radius, $30^{\circ}$ cone angle cantilever tip (MicroMasch, NSC16).

Figure 2(a) shows an AFM image of an ablation crater obtained positioning the sample at the FZP first diffraction-order focal plane. This ablation crater and all the others shown in this work were produced with a single laser pulse. The crater was obtained attenuating the beam by $\sim 36 \times$, introducing 110 mTorr of Ar into the processing chamber. Attenuation of the laser beam results in a reduction of the crater diameter and depth [Fig. 2(b)]. The $230 \mathrm{~nm}$ diameter of the craters ablated with the attenuated laser beam matches the first-order diffraction spatial resolution of the FZP. The depth of the craters ablated without attenuation is $250 \mathrm{~nm}$, which is significantly larger than the $19 \mathrm{~nm}$ attenuation length of the $46.9 \mathrm{~nm}$ light in PMMA. ${ }^{11}$ The created plasma, with an estimated temperature of $\mathrm{Te}=1-3 \mathrm{eV}$, has an acoustic velocity that is $\sim 10 \times$ larger than that necessary to clear the hole within the laser pulse duration. The shot-to-shot reproducibility of the ablation was found to be very good, as illustrated by the AFM image in Fig. 3. The edges of the craters are very abrupt and their walls are very smooth. The generation of high-quality ablated surfaces is assisted by the strong localization of the absorbed energy, i.e., the attenuation and thermal diffusion lengths here are very short, and by the prevalence of chain scissions at short wavelengths. ${ }^{11,12}$

Smaller craters can in principle be obtained using the tighter third-order focus of the zone plate. Figure 4 shows cross sections of the ablation craters obtained with the third diffraction order, which contains only $\sim 1 / 10$ of the pulse energy in the firstorder focus. The crater created without attenuation has a diameter of $330 \mathrm{~nm}$ and a depth of $\sim 100 \mathrm{~nm}$,

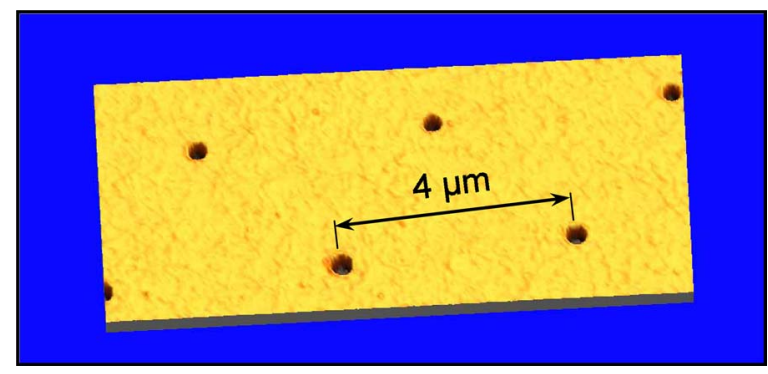

Fig. 3. (Color online) AFM image of a set of consecutive ablation craters showing good shot-to-shot reproducibility. The top row of the craters was obtained with larger beam attenuation.

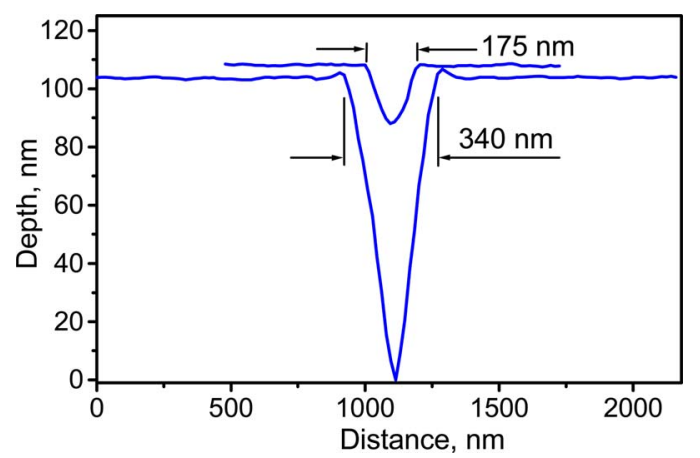

Fig. 4. (Color online) Cross sections of the ablation craters obtained with the third-order focus of the zone plate and attenuation factors of $1 \times$ (lower trace) and $5 \times$ (upper trace). 


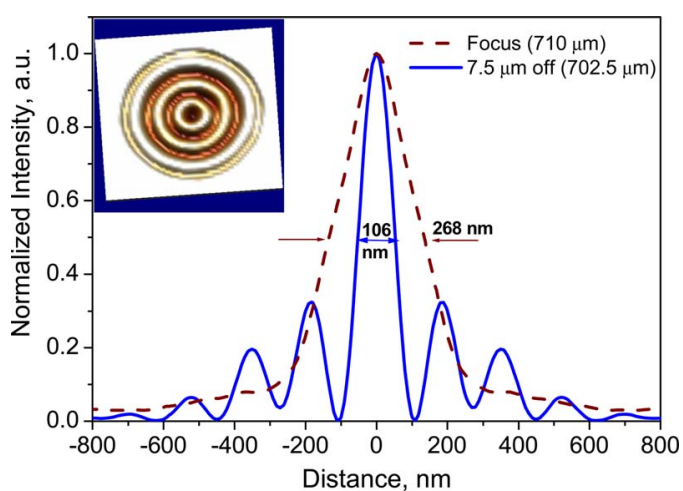

Fig. 5. (Color online) Calculated diffraction pattern at the third-order focal plane, $710 \mu \mathrm{m}$ from the FZP (dashed curve), and at $7.5 \mu \mathrm{m}$ away from it (solid curve). The broad intensity distribution at the focal plane arises from spherical aberrations. The central peak in the intensity distribution has a FWHM of $268 \mathrm{~nm}$ at the focal plane and narrows down to $106 \mathrm{~nm}, 7.5 \mu \mathrm{m}$ away. The inset shows an AFM image of a typical ablation pattern obtained outside of the focal plane without attenuation.

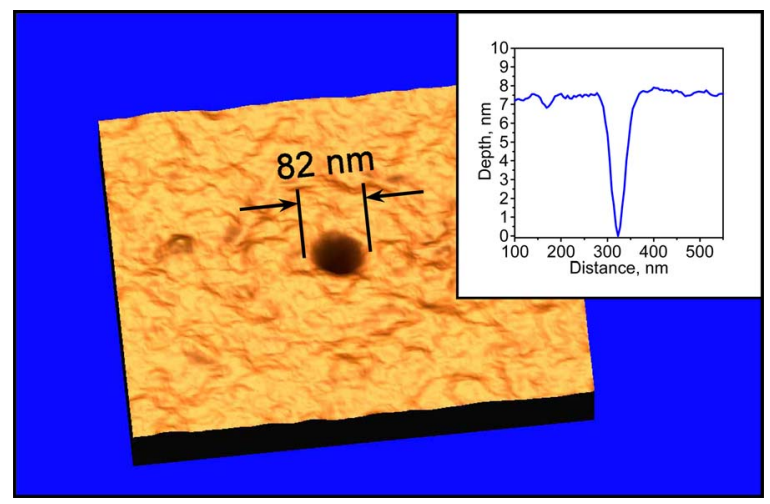

Fig. 6. (Color online) AFM image of an $82 \mathrm{~nm}$ diameter crater obtained placing the sample $\sim 7 \mu \mathrm{m}$ away from the third diffraction-order focal plane. The inset shows the crater's cross section.

and the crater formed with the laser beam attenuated by $\sim 5 \times$ is $170 \mathrm{~nm}$ wide and $16 \mathrm{~nm}$ deep. As in the case of first-order focusing, the craters have very clean walls. Nevertheless, the crater's diameters are significantly larger than could be expected from the resolution of a FZP in third-order operation $(\sim 80 \mathrm{~nm})$. This can be explained as the FZP used in the experiment was corrected for aberrations in the first-order focus but not in the third order. Thus the uncorrected spherical aberration causes the thirdorder diffraction focal spot to be wider. This was confirmed by simulating the intensity distribution at the third-order focal plane using the RayleighSommerfeld diffraction integral, ${ }^{13}$ which results in a peak with FWHM of $\sim 270 \mathrm{~nm}$ (Fig. 5). The simula- tion also showed that a much narrower intensity peak of $\sim 100 \mathrm{~nm}$ FWHM surrounded by concentric rings of decreasing intensity can be obtained at locations several micrometers away from the focal plane (e.g., $\sim 7.5 \mu \mathrm{m}$ ). This suggests that, by attenuating the intensity of the rings to values below the ablation threshold, it should be possible to use this narrow central peak to produce smaller craters. Craters with a diameter of $\sim 82 \mathrm{~nm}$ and a depth of $\sim 8 \mathrm{~nm}$ were obtained by placing the sample at $\sim 7 \mu \mathrm{m}$ away from the third-order focal plane (Fig. 6) and attenuating the beam by a factor of $\sim 5 \times$. These are, to the best of our knowledge, the smallest ablation craters obtained by directly focusing a laser beam with a lens onto a sample surface. The use of a FZP with a smaller outer zone width should produce holes of even smaller dimensions.

In summary, the results demonstrate the feasibility of directly patterning sub-100 $\mathrm{nm}$ features using a focused soft x-ray laser beam, opening a path for the development of new nanoprobes and nanomachining tools.

This work was supported by the Engineering Research Centers Program of the National Science Foundation under NSF award EEC-0310717. C. S. Menoni's e-mail address is carmen @engr.colostate.edu.

\section{References}

1. I. Avrutsky, D. G. Georgiev, D. Frankstein, G. Auner, and G. Newaz, Appl. Phys. Lett. 84, 2391 (2004).

2. P. P. Pronko, S. K. Dutta, J. Squier, J. V. Rudd, D. Du, and G. Mourou, Opt. Commun. 114, 106 (1995).

3. F. Korte, J. Serbin, J. Koch, A. Egbert, C. Fallnich, A. Ostendorf, and B. N. Chichkov, Appl. Phys. A 77, 229 (2003).

4. P. Simon and J. Ihlemann, Appl. Surf. Sci. 109/110, 25 (1997).

5. A. Chimmalgi, C. P. Grigoropoulos, and K. Komvopoulos, J. Appl. Phys. 97, 104319 (2005).

6. G. Wysocki, J. Heitz, and D. Bauerle, Appl. Phys. Lett. 84, 2025 (2004).

7. B. R. Benware, C. D. Macchietto, C. H. Moreno, and J. J. Rocca, Phys. Rev. Lett. 81, 5804 (1998).

8. C. D. Macchietto, B. R. Benware, and J. J. Rocca, Opt. Lett. 24, 1115 (1999).

9. E. H. Anderson, IEEE J. Quantum Electron. 42, 27 (2006).

10. D. T. Attwood, Soft X-Rays and Extreme Ultraviolet Radiation (Cambridge U. Press, 1999).

11. B. L. Henke, E. M. Gullikson, and J. C. Davis, At. Data Nucl. Data Tables 54, 181 (1993).

12. L. Juha, M. Bittner, D. Chvostova, J. Krasa, Z. Otcenasek, A. R. Präg, J. Ullschmied, Z. Pientka, J. Krzywinski, J. B. Pelka, A. Wawro, M. E. Grisham, G. Vaschenko, C. S. Menoni, and J. J. Rocca, Appl. Phys. Lett. 86, 034109 (2005).

13. Q. Cao and J. Jahns, J. Opt. Soc. Am. A 21, 561 (2004). 